\title{
The Mesenteric Lipoma in Children
}

ISSN: 2576-9200

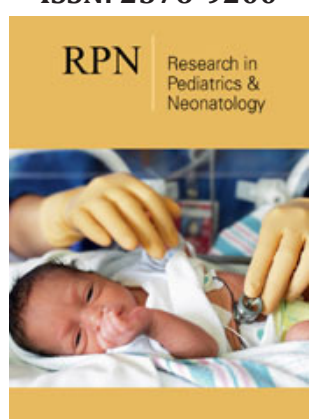

*Corresponding author: Hanine D, Pediatric Surgery Department "A", Children's Hospital of RABAT, Faculty of Medicine and Pharmacy, Mohammed V University Rabat, Morocco

Submission: 眥 April 01, 2021

Published: 制April 29, 2021

Volume 5 - Issue 4

How to cite this article: Hanine $D$, Rouijel B, El Baoudi A, Aboulam Z, Kisra M. The Mesenteric Lipoma in Children. Research in Pediatrics \& Neonatology. 5(4). RPN. 000616. 2021.

DOI: $10.31031 / R P N .2021 .05 .000616$

Copyright $\left(C_{C}\right.$ Hanine D. This article is distributed under the terms of the Creative Commons Attribution 4.0 International License, which permits unrestricted use and redistribution provided that the original author and source are credited.

\author{
Hanine $\mathrm{D}^{1,2 *}$, Rouijel B ${ }^{1,2}$, El Baoudi $\mathrm{A}^{1,2}$, Aboulam $\mathrm{Z}^{1,2}$ and Kisra $\mathbf{M}^{1,2}$ \\ ${ }^{1}$ Pediatric Surgery Department “A”, Children's Hospital of RABAT, Morocco \\ ${ }^{2}$ Faculty of Medicine and Pharmacy, Mohammed V University Rabat, Morocco
}

\section{Abstract}

We report the observation of a 5-year-old boy who consults for acute paroxysmal abdominal pain located in the right iliac fossa without other associated signs. The physical examination was completely normal.

Abdominal ultrasound showed a multi-compartmentalized cystic mass, without vascular character on Doppler, without mass effect on neighboring structures; the mesenteric vessels were in the normal position.

On computed tomography this mass corresponded to a mesenteric formation attached to the cecum containing several septas. The diagnosis of cystic lymphangioma or mesenteric lipoma was made. Surgical exploration confirmed the diagnosis of mesenteric lipoma attached to the ileum. Resection with anastomosis was performed. The post-operative consequences were simple. Pathological examination confirmed the lipomatous nature of the mass. At the end of this observation, we highlight this rare pathology with possible complications.

Keywords: Lipoma; Mesentery; Child

\section{Introduction}

Lipoma is a benign tumor made up of fatty tissue that usually does not cause complications [1]. Its mesenteric location is rare. Complications from mesenteric lipoma are exceptional, but remain possible, hence the importance of knowing them [2]. We report this condition in relation to a case in order to highlight its epidemiological, diagnostic and therapeutic aspects.

\section{Materials \& Methods}

Our study was done in Marsh 2021 at the pediatric surgery department "A" at the Children's Hospital of Rabat, the case studied is a 5-year-old boy brought by her parents in a table of abdominal pain from the right iliac fossa. Having as history, recurrent abdominal pain, often in the right iliac fossa and periumbilical, without fever, vomiting, rectal bleeding or transit disorders. Clinical examination shows a child in good general condition, of normal build, with well-colored conjunctivae. The abdominal palpation as well as the rest of the clinical examination is unremarkable.

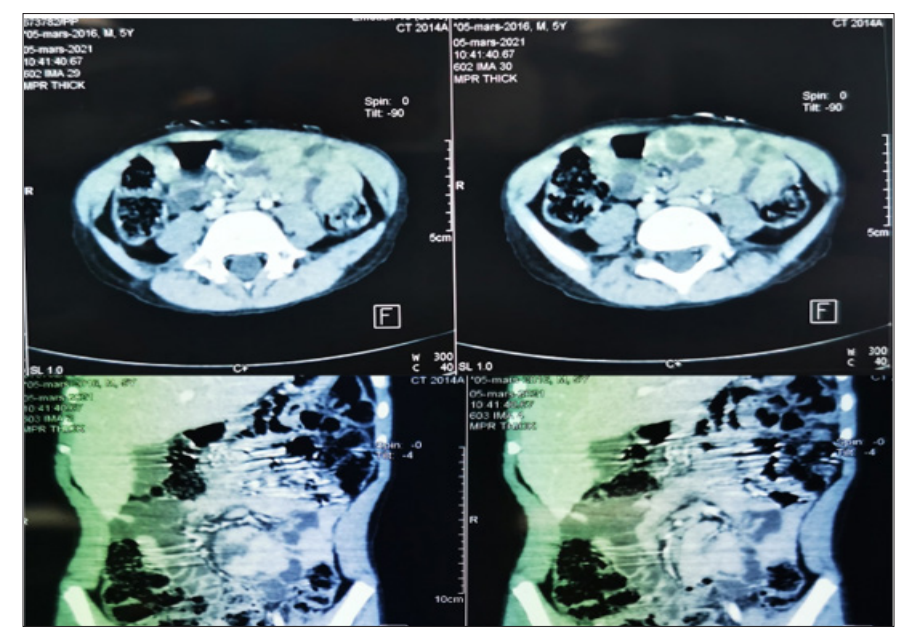

Figure 1: CT image showing a multi-compartmental mass between the digestive loops. 
A multitude of clinical diagnoses, both medical and surgical, have been mentioned. The laboratory tests were normal. X-ray examinations were performed to support the etiological diagnosis. An ASP was carried out without anomalies. Abdominal ultrasound, coupled with Doppler, showed a cystic, multi-compartmental mass of the right iliac fossa, devoid of vascularity, with normally located mesenteric vessels. Abdominal tomodensitometry objectified the presence of a voluminous oblong formation, mesenteric, attached to the cecal wall and to the right colon, insinuating between the digestive structures without pushing them back, of fluid density without clean wall, not enhanced after injection of the product. of contrast; first suspecting a cystic lymphangioma (Figure 1). We performed a laparotomy by a transverse incision at the Mc Burney point, after exploration of the ileocaecal intersection which is unremarkable.

The entire small intestine was unrolled, a mass of macroscopically lipomatous nature was found in the ileum $50 \mathrm{~cm}$ from the ileocaecal junction. This mass originates in the mesentery, and extends on either side of the latter by joining the digestive loop without a clear cleavage plane (Figures $2 \& 3$ ). The 2 layers of the mesentery are opened with total resection of the mass as well as the digestive loop to which it attaches (Figure 4), then end-to-end anastomosis and closure of the mesenteric breach. No other associated anomalies during exploration. The post-operative consequences were simple. Pathological examination concluded that lipomatous tissue was formed without signs of malignancy.

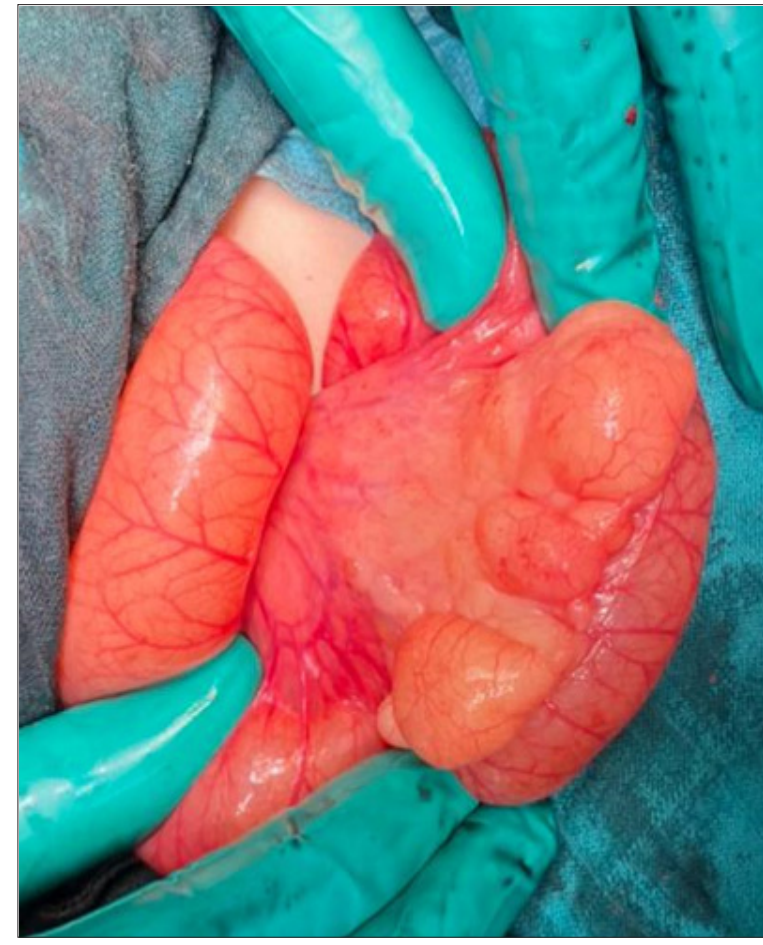

Figure 2: Surgical exploration showing the anterior surface of the mass.

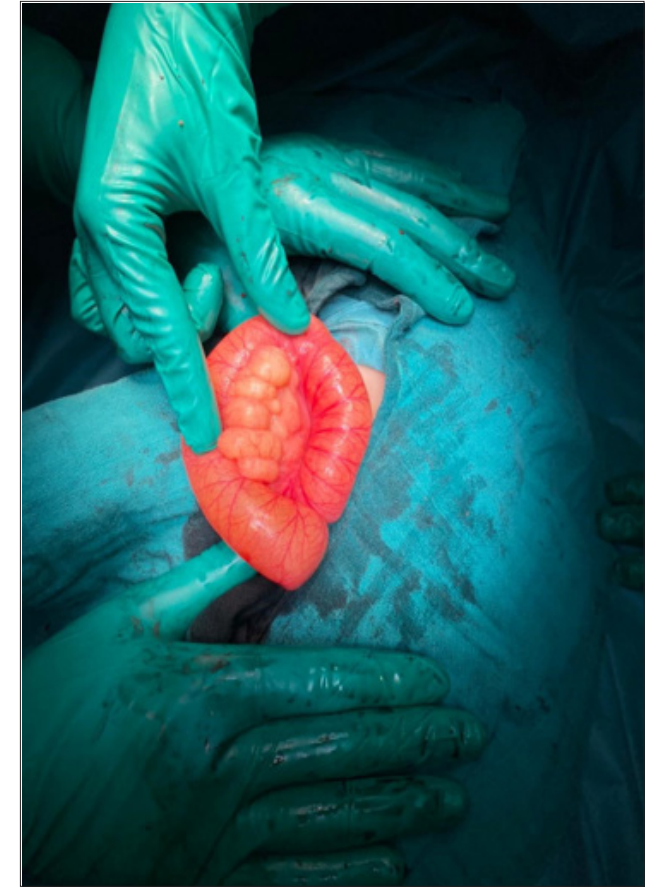

Figure 3: Surgical exploration showing the posterior surface of the mass.

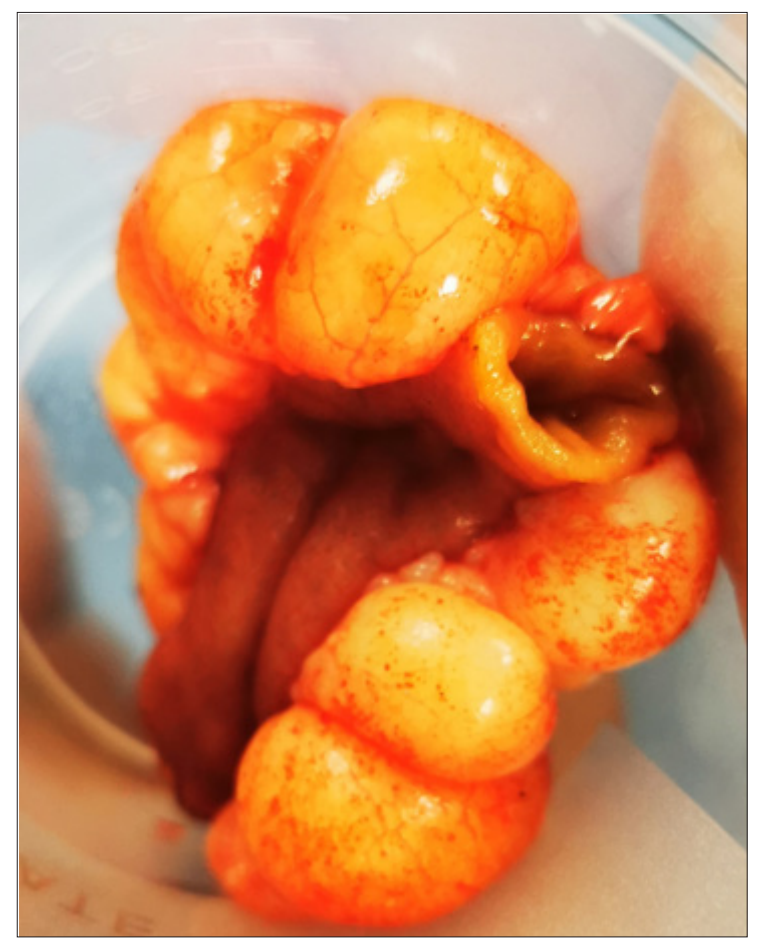

Figure 4: Resected lipomatous mass carrying the digestive loop.

\section{Discussion}

Lipoma is a benign tumor made up of fatty tissue that usually does not cause complications [1]. In children, lipomas are superficial 
in the trunk. Deep lipomas can involve the thorax, the mediastinum and rarely the intestinal mesentery [3]. A positive diagnosis of mesentery lipoma is often made at the time of laparotomy, as the clinical symptoms are very polymorphic and nonspecific $[3,4]$. It can be asymptomatic, discovered by chance, or manifested by an abdominal mass with digestive signs giving rise to fear of a major complication such as occlusive compression syndrome or volvulus $[4,5]$. Current radiological investigations, including ultrasound and computed tomography, are an important part of the diagnosis. However, other types of fatty tissue tumors such as liposarcoma cannot be formally ruled out until there is histological evidence $[3,5]$.

Ultrasound coupled with Doppler is an important contribution to highlight the mass when it is large or complications, namely a small bowel volvulus and its turns [6]. In addition, it makes it possible to evoke a suffering of the digestive loops in front of the thickening of the wall of the handles at the level of the volvulus $[6,7]$. Finally, this examination is easy to perform, non-invasive and painless [1]. In our patient, ultrasound revealed an avascular tissue mass, the nature of which has not been specified without any effect of mass on neighboring structures.

The study carried out by Kisra M \& Sarda [8,9] has shown that ultrasound is not always efficient, because the boundaries of the tumor are imprecise and the exact location in relation to the peritoneum is difficult to specify with precision. Likewise echogenicity is variable from one lipoma to another. This is the same thing for our patient. Computed tomography coupled with ultrasound can support the diagnosis of mesentery lipoma by studying the density of the tumor, its fatty nature, its exact location, size and extent $[1,9,10]$. In addition, it accurately determines the relationship with neighboring bodies. It also has the advantage of eliminating certain differential diagnoses, in particular teratoma by the absence of calcifications. The mesentery lipoma presents on the CT plan as an intraperitoneal mass, encapsulated, of fatty density, containing thin spans not enhanced by the product of the contrast, unrelated to the neighboring organs, while specifying its size $[3,11]$. In our patient, computed tomography did not allow us to retain the diagnosis by highlighting a partitioned cystic mass without confirming the lipomatous nature of the mass.

Surgical treatment is currently the treatment of choice for mesentery lipoma [6]. Two methods are described: classical laparotomy and laparoscopy which constitutes the gold standard in this pathology.

This treatment constitutes the most important time by making it possible, on the one hand, to prejudge the nature of the tumor and its macroscopic appearance, and on the other hand to confirm the diagnosis by pathological examination of the excision piece [6]. The diagnosis of a mesentery lipoma is most often made at the time of laparotomy. In children, the transverse incision is the most used $[3,8]$ as was the case for our patient.

Complete excision is the highlight of surgical management in the absence of intestinal necrosis. This resection prevents sarcomatous degeneration and other complications, namely volvulus [11]. In our patient we performed a transverse laparotomy at McBurney's point, an excision of the mass by removing the digestive lumen by endto-end resection-anastomosis after release of the meso, because the mass surrounded the digestive loop on both sides. other of the meso. The anatomopathological study made it possible to confirm the histological diagnosis.

\section{Conclusion}

Lipoma of the mesentery is a rare benign condition in children. It mainly affects the mesentery of the small intestine. Clinically, it is either asymptomatic or revealed during a non-specific symptomatology or a complication such as small bowel volvulus. Ultrasound is not always reliable to make the diagnosis, hence the need to perform a CT scan. Its treatment remains surgical.

\section{References}

1. Ozel SK, Apak S, Ozercan IH, Kazez A (2009) Giant mesenteric lipoma as a rare. J Korean Med Sci 24(2): 333-336.

2. Cherian A, Singh SJ, Broderick N, Zaitoun AM, Kapila L (2004) Small bowel volvulus due to giant mesenteric lipoma. Pediatr Surg Int 20(1112): 869-871.

3. Lambot K, Lougue-Sorgho LC, Gorincour G, Chapuy S, Chaumoitre K, et al. (2005) Non-traumatic abdominal emergencies in pediatric practice. J Radiol 86(2 Pt 2): 223-233.

4. Livne PM, Zer M, Shmuter Z, Dintsman M (1981) Acute intestinal obstruction caused by necrotic mesenteric lipoma: A case report. Am J Proctol Gastroenterol Colon Rectal Surg 32(11): 19-22.

5. Kaniklides C, Frykberg T, Lundkvist K (1998) Pediatric mesenteric lipoma, an unusual cause of repeated abdominal pain. Acta Radiol 39(2): 695-697.

6. McCoubrey AS, Thompson RLE (2006) Small bowel volvulus secondary to a mesenteric lipoma: A case report and review of the literature. Ir J Med Sci 175(4): 79-80.

7. Rezvanizadeh A, Mohammad SS, Mehrzad M, Alizadeh H (2013) Volvulus caused by mesenteric lipoma. Iran J Pediatr 23(1): 121-123.

8. Kisra M, Ettayeby F, Zerhouni H, Benhammou M (2003) Interest of laparoscopy in the management of mesentery lipoma. The Journal of Coelio-Chirurgie 41(2): 75-77.

9. Prando A, Wallace S, Marins JL, Pereira RM, de Oliveria ER (1998) Sonographic features of benign intraperitoneal lipomatous tumors in children-report of 4 cases. Pedatr Radiol 20(8): 571-574.

10. Sarda H, Loc'h HL, Lucas G, Gaulier A, Lehujeur C (1992) Lipomas of the mesentery in young children. Ann Pédiatr(Paris) 39(10): 633-634.

11. Sato M, Ishida A, Konno K, Komatsuda T, Naganuma H, et al. (2002) Mesenteric lipoma: Report of a case with emphasis on US findings.Euro Radiol 12(4): 793-795. 